\title{
Predicting the coherence resonance curve using a semi-analytical treatment
}

\author{
Santidan Biswas * Dibyendu Das, P. Parmananda, and Anirban Sain \\ Department of Physics, Indian Institute of Technology Bombay, Powai, Mumbai-400 076, India.
}

(Dated: September 13, 2021)

\begin{abstract}
Emergence of noise induced regularity or Coherence Resonance in nonlinear excitable systems is well known. We explain theoretically why the normalized variance $\left(V_{N}\right)$ of inter spike time intervals, which is a measure of regularity in such systems, has a unimodal profile. Our semi-analytic treatment of the associated spiking process produces a general yet simple formula for $V_{N}$, which we show is in very good agreement with numerics in two test cases, namely the FitzHugh-Nagumo model and the Chemical Oscillator model.
\end{abstract}

PACS numbers: 05.45.-a, 02.50.-r, 05.40.-a

\section{INTRODUCTION}

Many deterministic, nonlinear, excitable systems, for example, the FitzHugh-Nagumo model (FHN) [1] or the Chemical Oscillator model (CO) [2], undergo bifurcation from a stable focus to a stable limit cycle (LC) behavior when a system parameter is tuned. However, holding the parameter near the bifurcation point, on the stable focus side the system can still be made to exhibit spiking behavior (which is otherwise the signature of a limit cycle), by adding a random uncorrelated noise to the system. The noise forces the system to intermittently jump across the bifurcation point in the parameter space. As a result of these random excursions, the system exhibits intermittent cyclic behavior which manifests as spikes in the dynamical variable. Interestingly, the time intervals $\tau_{p}$, between two successive noise driven spikes, which are in general irregular, strangely becomes fairly regular at an optimal noise value (the resonance point). This phenomenon is called Coherence Resonance. It has attracted considerable interest theoretically as well as experimentally $[3,4,5,5,6,7,8,8,9,10]$, as quite counter-intuitively order arises with the aid of tuned randomness. A quantitative means of detecting this resonance point is enumerating the normalized variance $\left(V_{N}\right)$ defined by $V_{N}=\sqrt{\left\langle\tau_{p}^{2}\right\rangle-\left\langle\tau_{p}\right\rangle^{2}} /\left\langle\tau_{p}\right\rangle$, as a function of noise strength. Here $\langle$.$\rangle denotes statistical time average. Typically V_{N}$ is enumerated from time-series analysis of spikes generated by the system, subjected to noise. The noise strength at which minimum of $V_{N}$ occurs is the desired point of resonance.

The analytical work so far on this subject, have either dealt with a toy model [1], or addressed special limits of the FHN model e.g, very weak noise [11], and infinite time scale separation between the fast and slow variables [9, 12, 13]. A pioneering qualitative understanding of the phenomenon is given by Pikovsky and Kurths [1], who argue that the resonance happens as a competition between two time scales - the activation time $t_{a}$ (the time between the end of one spike and beginning of another) and the excursion time $t_{e}$ i.e., duration of a spike. The inter spike interval (ISI) $\tau_{p}=t_{a}+t_{e}$. They claim that $t_{a}$ has a strong dependence on noise intensity and follows a simple Kramer's [14] like formula, whereas $t_{e}$ has a much weaker noise dependence and corresponds to the decay time of unstable excited state. Kramers theory describes the noise driven escape time $\tau_{\text {esc }}$ of a particle (say $y$ ) from a deep potential trap, and gives $\tau_{\text {esc }} \sim \exp \left(E_{b} / D^{2}\right)$; here $D$ is noise amplitude, and $E_{b}$ is the barrier height. But excitable systems with two coupled variables $x$ and $y$ pose new challenges: the barrier $E_{b}$ is both dynamic and $D$ dependent. The effective barrier for $y$ is dynamic as it is generated by $x$ which itself is a dynamical variable. Furthermore our numerical studies show that barrier parameters, like its width $\delta$, are indeed $D$ dependent. In this paper, we avoid invoking Kramers picture apriori, and show that the timescales $t_{e}$ and $t_{a}$ can be understood from alternative arguments.

We derive below a simple theoretical formula for $V_{N}$, which will be generally applicable to any nonlinear system exhibiting coherence resonance. There are parameters in the universal formula, which depend on the specific details of the nonlinear system at hand, and can only be fixed by some amount of numerical or alternatively experimental analysis. Thus the formula is semi-theoretical. Although this may seem as no less work than the usual time-series analysis, as we show below, it certainly involves incorporation of enhanced understanding of the phenomenon compared to what existed before. To support our claim of generality, we study two very different nonlinear systems: the FHN model [1] and the CO model [2, 10, 15]. We show that our predicted formula fits quite well, with the curve of $V_{N}$ obtained by brute force time-series analysis, in both the cases.

\section{MODEL}

Before starting our main analysis, let us define the FHN and $\mathrm{CO}$ systems in the presence of noise, to make this paper self contained. The FHN model has the following equations

$$
\epsilon \frac{d x}{d t}=x-\frac{x^{3}}{3}-y, \quad \frac{d y}{d t}=x+a+D \xi(t) .
$$

Here $a, D$ and $\epsilon(\ll 1)$ are the three parameters. For $|a|>1$, there is a stable fixed point at $x_{*}=-a, y_{*}=\frac{a^{3}}{3}-a$, while for $|a|<1$ a limit cycle exists in the $x-y$ space and dynamics of both the variables are periodic. The value of $a$ on the fixed point side, which we hold fixed for our simulation, is denoted by $a_{0}$. The parameter $D$ is the amplitude of the Gaussian white noise $\xi$, for which $\langle\xi(t)\rangle=0$ and 


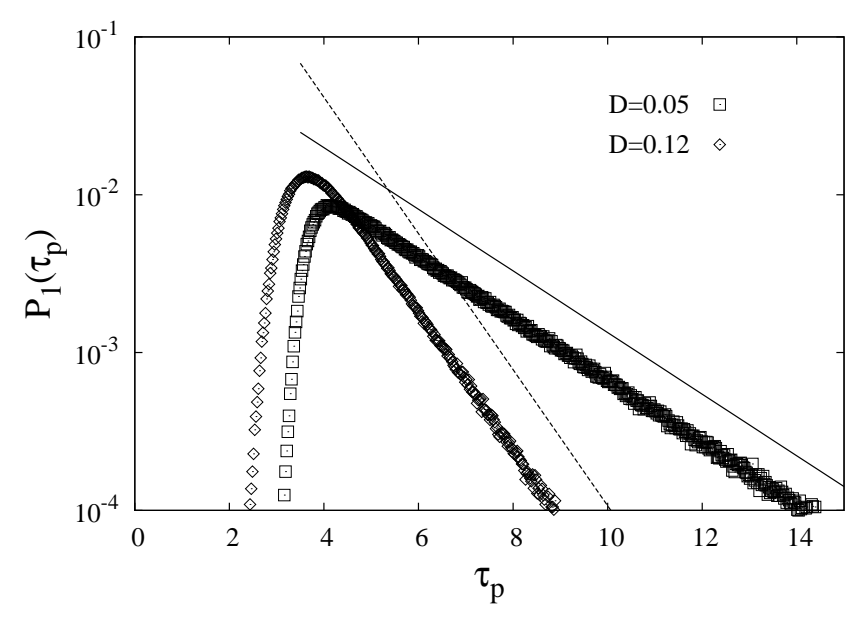

FIG. 1: Semi-log plot of $P_{1}\left(\tau_{p}\right)$ against $\tau_{p}$ for FHN model. Here $a_{0}=1.1$ and $\epsilon=0.01$ (see Eq. (11) - these same values are used for other figures in the paper. The straight lines are exponential fits (placed higher for visual clarity) to the tail to obtain $t_{\mathrm{esc}}^{\text {num }}$ values and they are $2.225,1.007$ for $D=0.05$ and 0.12 respectively. The corresponding $t_{\min }^{\text {num }}$ (defined in the text) values are 3.573 and 2.904 .

$\left\langle\xi(t) \xi\left(t^{\prime}\right)\right\rangle=\delta\left(t-t^{\prime}\right)$. The small parameter $\epsilon$ makes the motion on the limit cycle much faster along the $x$ direction than the $y$. The second model of $\mathrm{CO}$ is defined by the following equations:

$$
\begin{aligned}
\epsilon \frac{d u}{d t} & =\frac{v-u}{R}-f(u, c), \\
\frac{d c}{d t} & =\frac{u-v}{R}+(1-c)+\alpha f(u, c)
\end{aligned}
$$

where $f(u, c)=c\left(a_{1} u+a_{2} u^{2}+a_{3} u^{3}\right)$ and $v=v_{0}+D \xi(t)$. Here $u$ and $c$ are the dynamical variables and $R, v_{0}, a_{1}, a_{2}$, $a_{3}, \epsilon, \alpha$, and $D$ are the parameters. $v$ is the bifurcation parameter. Limit cycle exists for the values $v \leq 29.235$ whereas for $v>29.235$, a steady state fixed point behavior is observed. The system variables and parameters are derived from the reaction-rate kinetics of the interacting chemical species. The details regarding the construction of the model equation are furnished elsewhere [2, 10, 15].

\section{RESULTS}

If one makes a simple-minded first guess that the inter spike intervals $\tau_{p}$ have a Poisson distribution, then $V_{N}$ would be a constant (independent of noise strength) which is empirically not the case. So what is the distribution of $\tau_{p}$ ? For a random train of spikes which are almost independent, it seems very likely that the distribution of ISI will have an exponential tail [16]. Yet a specialty of the spikes in the non-linear systems of our concern, is that a new spike cannot arise until the last spike subsides. Thus $\tau_{p}$ cannot be any smaller than characteristic 'spike width' $w_{s}$ (a finite quantity), i.e. the distribution of $\tau_{p}$ is expected to have a sharp lower cutoff at some finite $\tau_{\min }$.
We stress here that if this lower cutoff were absent, then $V_{N}$ would have had no variation and coherence resonance would have vanished. Thus we expect the probability density of $\tau_{p}$ to be,

$$
P_{1}\left(\tau_{p}\right)=N \Theta\left(\tau_{p}-\tau_{\min }(D)\right) \exp \left(-\frac{\tau_{p}}{\tau_{\mathrm{esc}}(D)}\right) .
$$

Here $\Theta$ is the Heaviside function [17], while $\tau_{\text {esc }}$ is the characteristic time associated with exponential tail of $P_{1}\left(\tau_{p}\right)$. In Eq. (3), the normalization constant $N=\left(e^{\tau_{\min } / \tau_{\text {esc }}}\right) / \tau_{\text {esc }}$. We have checked that the distribution of $\tau_{p}$ obtained from the time series analysis of the FHN and CO models are consistent with Eq. (3) - see Fig. 1 for numerically obtained $P_{1}\left(\tau_{p}\right)$ for the FHN system for two different $D$ values. Despite the two values of $D$, one being away and another close to the resonance point, one can see clearly that the shape of the curves $P_{1}\left(\tau_{p}\right)$ shows no qualitative variation. Of course the quantities $\tau_{\min }^{\text {num }}$ and $\tau_{\text {esc }}^{\text {num }}$ (where the superscript "num" denotes numerical) are functions of $D$; in fact both decrease with $D$. The notational distinction between $\tau_{\min }^{\text {num }}$ in Fig. 1 and $\tau_{\min }$ in Eq. (3) is necessary, as the numerical curve in Fig. 1 does not rise strictly as a $\Theta$ function. To be precise, in Fig. 11 $\tau_{\min }^{\text {num }}$ is defined as the average of the time $\tau_{\text {min }, 1}^{\text {num }}$ at which $P_{1}\left(\tau_{p}\right)$ just starts becoming nonzero and the time $\tau_{\text {min, }}^{\text {num }}$ at which $P_{1}\left(\tau_{p}\right)$ reaches a peak. On the other hand $\tau_{\text {esc }}^{\text {num }}$ is obtained by fitting an exponential to the tail of $P_{1}\left(\tau_{p}\right)$. In this paper we attempt to obtain $\tau_{\min }$ and $\tau_{\text {esc }}$ theoretically, as opposed to the numerical estimates $\tau_{\min }^{\text {num }}$ and $\tau_{\text {esc }}^{\text {num }}$ just described. Note that the quantities $\tau_{\min }^{\text {num }}$ and $\tau_{\text {esc }}^{\text {num }}$ are analogous to the quantities $\tau_{e}$ and $\tau_{a}$ respectively as discussed in [1].

The first and the second moments of $\tau_{p}$, namely $\left\langle\tau_{p}\right\rangle$ and $\left\langle\tau_{p}^{2}\right\rangle$, can be easily obtained using Eq. (3) and using them in the definition of $V_{N}$ we get

$$
V_{N}=\frac{\tau_{\mathrm{esc}}(D)}{\tau_{\min }(D)+\tau_{\mathrm{esc}}(D)} .
$$

The simple formula for $V_{N}$ above, is the central result of this paper [18], and is a good approximation in general for any non-linear system exhibiting coherence resonance, provided one could predict $\tau_{\text {esc }}(D)$ and $\tau_{\min }(D)$ theoretically. In what follows we try to do the latter. A similar formula as Eq. (4) was derived, although in the low $D$ limit [11] for anticoherence resonance.

Formally, the resonance point is obtained by setting the derivative of $V_{N}$ w.r.t. $D$ equals 0 . That implies the following relation

$$
\tau_{\text {esc }}^{\prime}\left(D_{\text {res }}\right) \tau_{\min }\left(D_{\text {res }}\right)=\tau_{\text {esc }}\left(D_{\text {res }}\right) \tau_{\min }^{\prime}\left(D_{\text {res }}\right) .
$$

Here $D_{\text {res }}$ denotes the value of $\mathrm{D}$ at the minimum of the $V_{N}$ curve i.e. at the resonance point. $\tau_{\mathrm{esc}}^{\prime}\left(D_{\text {res }}\right)$ and $\tau_{\min }^{\prime}\left(D_{\text {res }}\right)$ denote their respective derivatives with $D$ evaluated at $D_{\text {res }}$ . However, since both $\tau_{\min }\left(D_{\text {res }}\right)$ and $\tau_{\text {esc }}\left(D_{\text {res }}\right)$ are system specific and are obtained partly numerically, the scope of the analytical application of Eq. (5) is limited. 

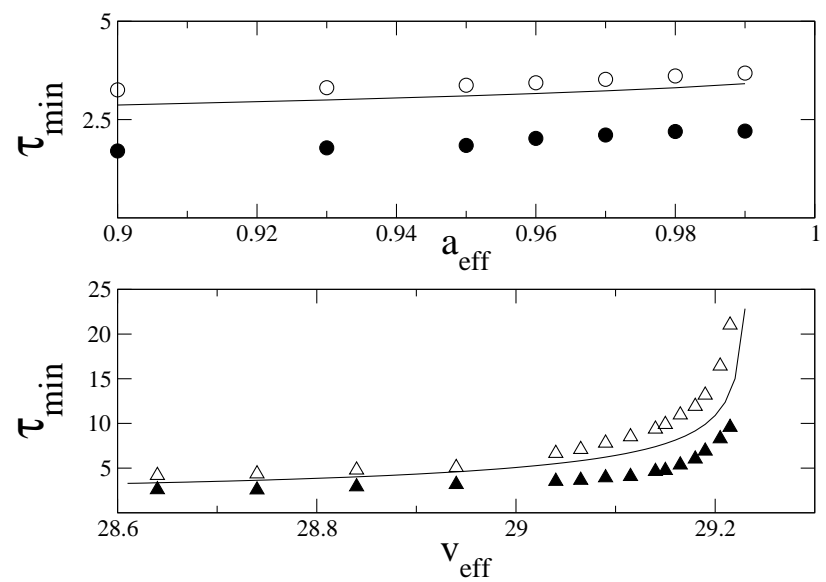

FIG. 2: Top frame: $\tau_{\min }(D)$ versus $a_{\mathrm{eff}}(D)$ in FHN. Bottom frame: $\tau_{\text {min }}(D)$ versus $v_{\text {eff }}(D)$ in CO. Here $v_{0}=29.24, \epsilon=0.03, \alpha=$ $0.1, a_{1}=1.125, a_{2}=-0.075, a_{3}=0.00125, R=10$ (see Eq. (2) - these same values are used for $\mathrm{CO}$ in other figures. For both frames: The empty symbols are for $\tau_{\mathrm{min}, \mathrm{r}}^{\mathrm{num}}$ and filled symbols are for $\tau_{\min , 1}^{\text {num }}$. The solid lines represent $\tau_{\min }$ from Eq. 6. The numerical data and the theoretical curves shows excellent agreement.

We start with a hypothesis about the functional dependence of $\tau_{\min }$ on $D$. We claim that the action of noise on Eq. (1) (or 2) merely shifts $a$ (or $v$ ) to $a_{\text {eff }}$ (or $v_{\text {eff }}$ ), with $a_{\text {eff }}=a_{0}-D$ (or $v_{\text {eff }}=v_{0}-D$ ). To be brief let us focus on the FHN system and the parameter $a$. The parameter value $a_{0}$ corresponds to the initial stable fixed point. The $a_{\text {eff }}$ makes the system feel that it is on the LC side, across the bifurcation threshold $a_{\mathrm{th}}=1$, and lead to a spike. The width of the spike $\tau_{\min }$ is expected to be equal to the time period of the effective LC experienced, say $t_{\mathrm{lcp}}$, i.e.,

$$
\tau_{\min }(D)=t_{\mathrm{lcp}}\left(a_{\mathrm{eff}}\right), \text { and analogously for } v .
$$

Here we assume that $t_{\mathrm{lcp}}$, which is the property of the system is known apriori as a function of $a$. Note that the system can spike even if $a_{\text {eff }}$ does not cross $a_{\mathrm{th}}$ (and $\tau_{\min }^{\text {num }}$ can be measured numerically), but our above claim is not valid as $t_{\mathrm{lcp}}$ is undefined. In the later case, we would claim that $\tau_{\min }(D)=w_{\mathrm{s}}$, the spike width.

We proceed to test Eq. (6) in FHN and CO models. In both the top (for FHN) and bottom (for CO) frames of Fig. 2, the solid lines are as per Eq. (6). Instead of plotting $\tau_{\min }^{\text {num }}$, for more clarity, we have plotted $\tau_{\text {min, }}^{\text {num }}$ in empty symbols and $\tau_{\min , 1}^{\text {num }}$ in filled symbols. The fact that $\tau_{\min }$ falls in between $\tau_{\min , \mathrm{r}}^{\mathrm{num}}$ and $\tau_{\min , 1}^{\mathrm{num}}$ for the range of $D$ studied, and the agreement being excellent for two distinct systems FHN and CO (with distinct $t_{\mathrm{lcp}}(a)$ and $t_{\mathrm{lcp}}(v)$ functions), gives strong empirical support for the formula in Eq. (6).

Next, we turn to $\tau_{\text {esc }}$ in Eq. (4). The dynamics of one of the variables in the non-linear system, for example $y$ in FHN or $c$ in $\mathrm{CO}$, under finite noise strength $D$, can be viewed as a stochastic process around the stable fixed points $y_{*}$ or $c_{*}$, respectively. For subsequent discussion we focus on FHN, but the results apply generally to any non-linear system exhibit-
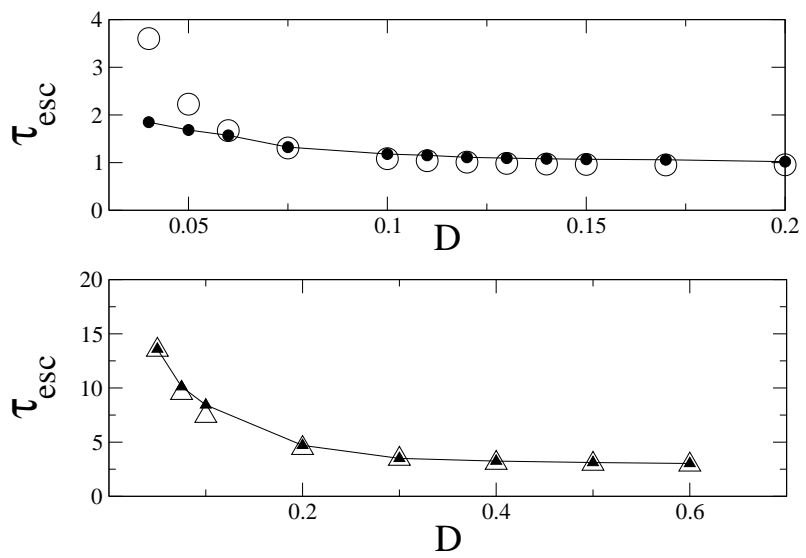

FIG. 3: $\tau_{\text {esc }}(D)$ versus $D$ for FHN (top frame) and CO (bottom frame) denoted by line joining filled symbols (the values of $\bar{\tau}_{0}(D)$ and $\delta_{m}(D)$ used for this plot are discussed in the text and fig. 4 Open symbols represents the numerical values $\tau_{\text {esc }}^{\text {num }}(D)$, obtained from fits as in fig 1

ing coherence resonance. Most often the noise displaces $y$ a little and then it relaxes back to the fixed point, in a typical excursion time $\overline{\tau_{0}}$. Occasionally however, if the excursion of the variable (e.g. $\Delta y=y-y_{*}$ in FHN) falls below a certain threshold denoted by a typical $-\delta_{m}$ (here $\delta_{m}>0$ ), the system exhibits a cycle and $y$ exhibits a spike. The latter amounts to absorption of $\Delta y$ at the boundary $-\delta_{m}$, on its first passage.

Specific system dependent details of the shape of the effective trapping potential is necessary to analytically calculate the above mentioned typical first passage time $\tau_{\text {esc }}$. Since our purpose is to remain as general as possible, we make a simplifying general assumption that after every random kick the relaxation is instantaneous. In effect this is equivalent to coarse-graining in time over units of the typical excursion time $\overline{\tau_{0}}$ (mentioned above and defined below).

Thus every excursion $\Delta y \neq 0$ at every discrete time step, may be treated as independent, and merely follows the noise and therefore has the same (Gaussian) distribution as the noise. Then it immediately follows, that the probability $Q(n)$ that the signal $\Delta y$ does not go below $-\delta_{m}$ for $n$ successive time steps and does so in the $(n+1)^{\text {th }}$ step is

$$
\begin{aligned}
Q(n) & =\left[P_{>}\left(-\delta_{m}\right)\right]^{n} P_{<}\left(-\delta_{m}\right) \\
& =e^{-n \ln \left(\frac{1}{P_{>}\left(-\delta_{m}\right)}\right)} P_{<}\left(-\delta_{m}\right)
\end{aligned}
$$

where $P_{>}(y)=\frac{1}{\sqrt{\pi D^{2}}} \int_{y}^{\infty} e^{-\frac{x^{\prime 2}}{D^{2}}} d x^{\prime}$ and $P_{<}=1-P_{>}$. Eq (7) shows that $Q(n)$ is exponential distributed, and its decay constant gives the "typical first passage time" $[16]$ in units of $\overline{\tau_{0}}$ :

$$
\begin{aligned}
\tau_{\text {esc }}(D) / \bar{\tau}_{0} & =-\left[\ln \left(P_{>}\left(-\delta_{m}\right)\right)\right]^{-1}, \quad \text { where } \\
P_{>}\left(-\delta_{m}\right) & =\left(1+\operatorname{erf}\left(\delta_{m} / D\right)\right) / 2
\end{aligned}
$$

and $\operatorname{erf}($.$) is the Error function [17].$

Note that the $D$ dependence of $\tau_{\text {esc }}$ comes from explicit dependence of $P_{>}$on $D$, as well as the implicit dependence 

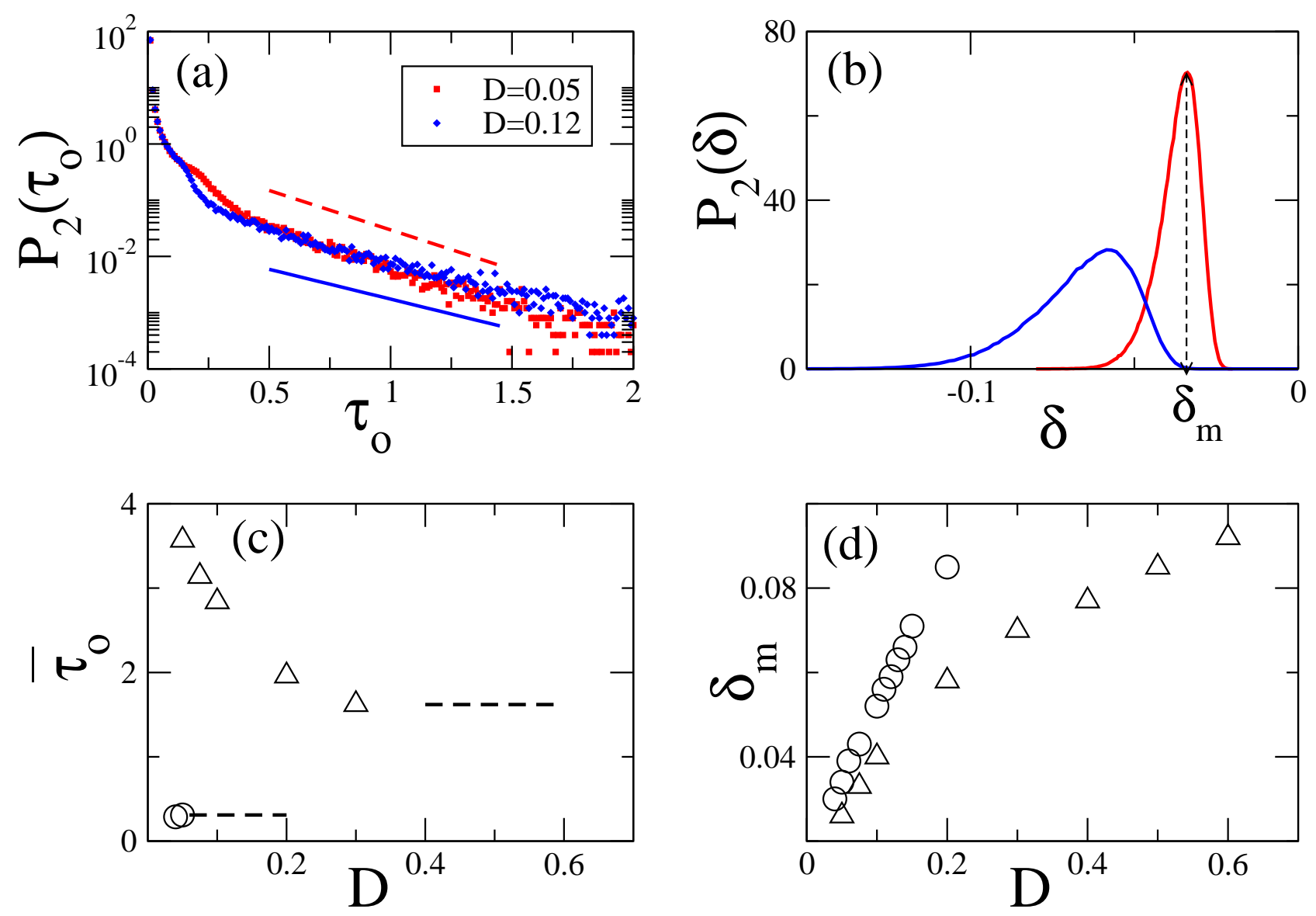

FIG. 4: (Color online) (a) PD of $\tau_{0}$ for the FHN along with exponential fit $e^{-t / \overline{\tau_{0}}}$ for two different $D$ values. (b) PD of $\delta$, and depiction of $\delta_{m}$ as most probable $\delta$ for the same two $D$ as in (a). (c) $\overline{\tau_{0}}$ versus $D$ for FHN (o) and CO ( $\triangle$ ) extracted from figure (a). The dashed line segments are explained in the text. (d) $\delta_{m}$ versus $D$ extracted from figure (b) - two sets are for FHN (o) and CO ( $\triangle$ ).

of the time unit $\bar{\tau}_{0}$ and barrier location $\delta_{m}$ on $D$. Of course $\bar{\tau}_{0}$ and $\delta_{m}$ will be system specific and incorporate the detail nature of the dynamic potential trap. The procedure to find $\bar{\tau}_{0}(D)$ and $\delta_{m}(D)$ will be discussed later. If we assume that the latter two quantities are known a priori then Eq. (8) maybe claimed to be a "theoretical" formula, and compared to the numerical values of $\tau_{\text {esc }}^{\text {num }}$ obtained as in Fig. 1 In Fig. 3 we see that the agreement between the theoretical formula and numerical data are excellent.

Using the asymptotic expansion of $\operatorname{erf}($.$) [17] in Eq. (8)$ we get $\tau_{\text {esc }} / \bar{\tau}_{0} \approx$ constant for $D / \delta_{m} \gg 1$ and $\approx e^{\delta_{m}^{2} / D^{2}}$ for $D / \delta_{m} \ll 1$. The latter behavior has been referred to as Kramer's formula for $\tau_{\text {esc }}[1,11$ ], but one needs to be careful - unlike the usual Kramer's escape time formula, $\delta_{m}$ is not the barrier height of the potential well but rather proportional to the width of the well.

What remains to be discussed is determination of $\overline{\tau_{0}}(D)$ and $\delta_{m}(D)$. To define $\overline{\tau_{0}}$ precisely, we note that between two successive spikes of $y$, the process $\Delta y$ (and $\Delta c$ for $\mathrm{CO}$ model) crosses zero several times. Let $\tau_{0}$ be the time interval between zero crossings of $\Delta y$ which is same as the excursion time mentioned earlier. A probability distribution (PD) of $\tau_{0}$ is then found for every $D$, and the PD has an exponential tail as shown in Fig. (4)a). We define the time constant of the latter exponential fit to be $\overline{\tau_{0}}(D)$. For FHN and CO systems the $\overline{\tau_{0}}(D)$ thus obtained are shown in Fig. 4(c). But with increasing $D$ the time stretches between two spikes become very small, making determination of $\overline{\tau_{0}}(D)$ unreliable due to poor statistics. So we took $\overline{\tau_{0}}(D)$ to be a constant (denoted by dashed line segments in Fig. 4(c)), for the $D$ values beyond which $\overline{\tau_{0}}(D)$ could not be reliably determined. A posteriori justification of the latter adhoc assumption for $\overline{\tau_{0}}$ lies in the successful agreement with numerical data of $\tau_{\text {esc }}(D)$ (see Fig. 3].

Next, we define $-\delta$ as the threshold of $\Delta y$ at which spiking occurs. Then the PD of $\delta$ for every $D$ can be computed (see Fig. 4(b)) and the most probable value may be identified as $\delta_{m}$. Plot of $\delta_{m}$ is shown against $D$ for both FHN and CO systems in Fig. 4(d). These values of $\delta_{m}$ were used to obtain the theoretical curve in Fig. 3

Finally, one can directly plot the $V_{N}$ from the theoretical formulas in Eqs. (4), (6), and (8) and compare it with numerical $V_{N}$ obtained from time series analysis (see Fig. 5). Both for $\mathrm{FHN}$ and $\mathrm{CO}$ the agreement is quite good and the locations 

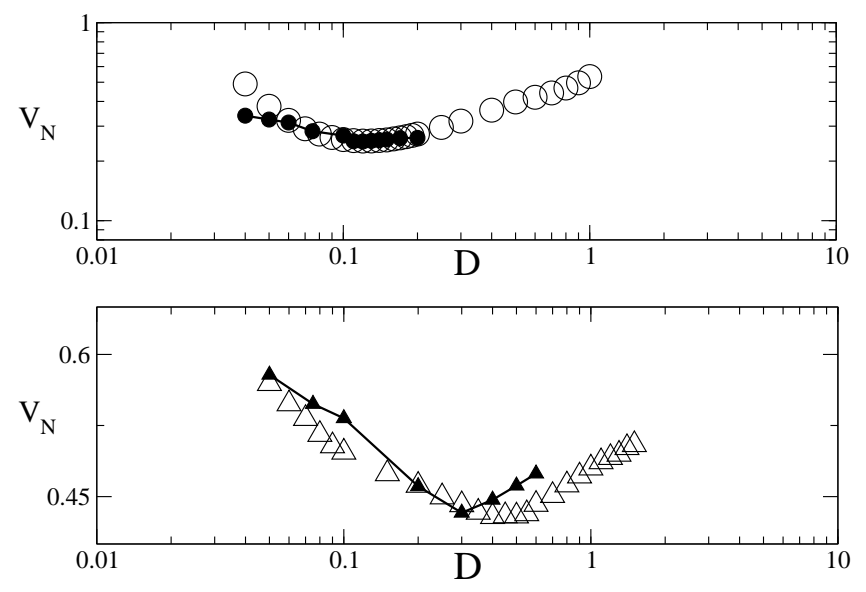

FIG. 5: Normalized Variance Plot for FHN(top) and CO(bottom). Open symbol denote the $V_{N}$ from numerical time series analysis. While, filled symbols joined by line represents the theoretical values from Eq. 44. The semi-analytic treatment was found to be valid for the following range of $D$ values: For the FHN model $0.04 \leq D \leq$ 0.2 . For the $\mathrm{CO}$ model $0.05 \leq D \leq 0.6$.

of resonance (the minima) are obtained within acceptable error limits.

\section{CONCLUSION}

Thus we claim to have found an alternate way of determining $V_{N}$ for non-linear systems exhibiting coherence resonance, based on theoretical considerations rather than brute force time series analysis. Only three empirical inputs are required for a specific system, namely (i) the limit cycle period $t_{\mathrm{lcp}}(a)$ as a function of the control parameter $a$, (ii) the typical zero-crossing time interval $\overline{\tau_{0}}(D)$ of the relevant stochastic dynamical variable, and (iii) the typical distance of excursion $\delta_{m}(D)$ beyond which the variable maybe regarded as "absorbed" (i.e. it spikes). We highlight the fact that the effective barrier parameters $\overline{\tau_{0}}$ and $\delta_{m}$ turn out to be $D$ dependent. It may seem no less work to obtain the empirical inputs (i)-(iii) for a system, yet once obtained they can be substituted in the simple theoretical formulas Eqs. (4), (6), and (8) and coherence resonance maybe predicted.

* Electronic address: santidan@ phy.iitb.ac.in

[1] A. Pikovsky and J. Kurths, Phys. Rev. Lett. 78, 775 (1997).

[2] A. Karantonis and S. Nakabayashi, Chem. Phys. Lett. 347, 133 (2001).

[3] R. Benzi, A. Sutera, and A. Vulpiani, J. Phys. A. 14, L453 (1981).

[4] R. Benzi, G. Parisi, A. Sutera, and A. Vulpiani, Tellus 34, 10 (1982).

[5] C. Nicolis and G. Nicolis, Tellus 33, 225 (1981).

[6] C. Nicolis, Tellus 34, 1 (1982).

[7] L. Gammaitoni, P. Hanggi, P. Jung, and F. Marchesoni, Rev. Mod. Phys. 70, 223 (1998), and the references within.

[8] A. Neiman, Scholarpedia 2, 1442 (2007).

[9] B. Lindner, J. García-Ojalvo, A. Neiman, and L. SchimanskyGeier, Phys. Rep. 392, 321 (2004).

[10] P. Parmananda, G. J. Escalera Santos, M. Rivera, and K. Showalter, Phys. Rev. E. 71, 031110 (2005).

[11] A. M. Lacasta, F. Sagués, and J. M. Sancho, Phys. Rev. E. 66, 045105(R) (2002).

[12] B. Lindner and L. Schimansky-Geier, Phys. Rev. E. 60, 7270 (1999).

[13] B. Lindner and L. Schimansky-Geier, Phys. Rev. E. 61, 6103 (2000).

[14] H. A. Kramers, Physica 7, 284 (1940).

[15] G. J. Escalera Santos, J. Escalona, and P. Parmananda, Phys. Rev. E 73, 042102 (2006).

[16] C. W. Gardiner, Handbook of Stochastic Methods for Physics, Chemistry, and the Natural Sciences (Springer-Verlag, Berlin, 1983).

[17] G. B. Arfken and H. J. Weber, Mathematical Methods for Physicists, 5th ed. (Harcourt Academic Press, 2001).

[18] Note that in Eq. (4) if $\tau_{\min }=0$, then $V_{N}=1$. 